to pertinent information by means of the alphabetical arrangement, a good index and an effective system of cross references. Entries are not in standard format but most contain a definition, a description of usage, an explication of the medico-moral issues involved and a discussion of the practical implications. References to published work are scanty but the dictionary is not intended as a bibliographic source. Some of the entries are masterpieces of succinct readable prose, and most are more than adequate but a few depart from the "noble gravity" which Dr Johnson required from a lexicographer.

For the purpose of this review I looked particularly at the handling of topics that have become highly profiled in recent years. I also took the stance of the Duchess in Alice in Wonderland: "Tut, tut,child! Everything's got a moral, if only you can find it". Firstly, "reproduction": the fundamental biology is precisely described and includes this sentence: "Reproduction in eutherian mammals also involves internal fertilisation and development (viviparity), the development of a chorio-allantoic placentation system, and the production of milk post-natally". Exactly so, and clear enough but associated ethical issues, as for instance; (i) the fetomaternal conflict produced by transplacental exposure to alcohol, heroin, and tobacco; (ii) placental insufficiency as major factor in coercive obstetric interference; (iii) the placenta as source of material for genetic diagnosis and for transplantation; (iv) ethics of breastfeeding and of the marketing of breastmilk substitutes, and (v) justification for the use of eutherian mammals in medical research, are missing.

In different style is the jokey statement: "English food has low fibre but Indian diet has high fibre. The former may lead to constipation but the latter to the "hurry with curry" syndrome". This light-hearted bit of popular gastroenterology appears in the entry on transcultural medicine, which also contains the now unacceptable categorisation of ethnicities into: Caucasian, Asian, Negroid, and Mongoloid (better discussions of ethnicity do occur elsewhere in the dictionary). There is no definition of "infant" despite the epidemiological, legal and philosophical significance of the term. The entry on hospital medicine makes no mention of mental or of Special Hospitals; the term traditional medicine is used differently in the entry on screening from the definitions given in that on traditional medicine.

Nowadays medical moral judgments are increasingly formulated by public opinion and media outrage which is widely disseminated but often poorly informed and has little regard for philosophical reflection, or balanced presentation of science and technology. The new dictionary acknowledges these shifts by excellent entries on medical journalism and on society, views in medical ethics but overall it comes over as an updated edition rather than a new work.

THOMAS E OPPE

Emeritus Professor of Paediatrics

\section{Volunteers in \\ Research and Testing}

Edited by Brony Close, Robert

Combes, Anthony Hubbard and John

Illingworth, London, Taylor and

Francis, 1997, 198 pages, $£ 45$.

I enjoyed this slim, well-edited conference proceedings! The editors did a fine job in selecting contributors who elucidated different aspects of the problem in 18 good brief chapters. Discussions of human volunteers are presented with emphasis on consumer and physician perspectives, existing law, special problems posed by the disadvantaged, and the role of ethics committees.

Several authors note that two factors dominate the motivation of volunteers: the need for money or the fear of an illness not well treated by current therapies. Thus, decision making is seldom autonomous as the consentee is intrinsically biased in favour of participation in the experiment.

This limits the absolute authority of informed consent and makes clear language very important. Any delusions I had about writing succinct clear English were nicely dispelled in Stanly Blenkinsop's chapter, "Whatever happened to plain English?" $\mathrm{He}$ repeatedly snipped and shortened, transforming paragraphs which seemed clear into the simpler and clearer.

Diminished autonomy lessens the distance between the mentally competent volunteer and the mentally diminished or imprisoned volunteer. All humans undergoing human experimentation must be protected:

The experiments must:
1. Be necessary,

2. Involve good science,

3. Have a favourable risk/benefit ratio, and

4. Provide data not obtainable by other means.

To ensure that these goals are met outside review is essential.

Which brings me to Douglas Smith's presentation of The Institute of Naval Research's system for protecting military personnel. The protocols and review procedures, with different levels of review for different degrees of uncertainty, are quite exceptional. While the nature of naval research is different from many sorts of innovative therapeutic and drug evaluations, the range of considerations in this fine chapter was gratifying. Because the risk to the volunteer is so often evident-let's see how well this suit protects you from this huge fire-the need for independent and dispassionate review of science, protocols and ethical matters are all required before the matter of consent can be broached.

Terrific model.

Moreover, as is evident throughout but explicitly in Michael Orme's chapter on risk, the risks of human experimentation are both small and considerable (at least 1:3,000 of a serious adverse event requiring hospitalisation). Good investigators are biased, at best because they believe in their experiments, at worst because ambition clouds judgment or diminishes concern for the patient.

Caution is essential. Good book.

MARK HILBERMAN, MD

The Carbondale Clinic, 1101 Village Road, Suite UL1A, Carbondale, Colorado 81623, USA

\section{The Making of the Unborn Patient: a Social Anatomy of Fetal Surgery}

Monica J Casper, New Brunswick, New Jersey, and London, Rutgers University Press, 1998, pages, $\$ 50$ hc, $\$ 20$ sc.

This book describes the development of interventions and surgical treatments for the fetus in the mother's womb. Such treatments, whether medical or surgical, performed to benefit the health of the fetus inevitably also affect the mother. The making 